\title{
Fractional and Integer Vortex Dynamics in Strongly Coupled Two-component Bose-Einstein Condensates from AdS/CFT Correspondence
}

\author{
Wei-Can Yang ${ }^{1}$, Chuan-Yin Xia ${ }^{2,1}$, Muneto Nitta ${ }^{3}$, and Hua-Bi Zeng ${ }^{1}$ \\ ${ }^{1}$ Center for Gravitation and Cosmology, College of Physical Science \\ and Technology, Yangzhou University, Yangzhou 225009, China \\ ${ }^{2}$ School of Science, Kunming University of Science and Technology, Kunming 650500, China and \\ ${ }^{3}$ Department of Physics, and Research and Education Center for Natural Sciences, \\ Keio University, Hiyoshi 4-1-1, Yokohama, Kanagawa 223-8521, Japan
}

\begin{abstract}
In order to study the rotating strongly coupled Bose-Einstein condensations(BEC), a holographic model defined in an AdS black hole that duals to a coupled two-component condensations in global $U(1)$ symmetry broken phase with intercomponent coupling $\eta$ and internal coherent coupling $\epsilon$ is proposed. By solving the dynamics of the model, we study the process of formation and also the crossover from fractional to integer vortex phases. With changing only $\eta$ from zero to a finite value, fractional vortex lattices undergo a transition from hexagon to square lattice and finally to vortex sheets. By continuing to turn on $\epsilon$, we find that two fractional vortices in different components constitute dimers, and when $\eta$ transcend a critical value, multi-dimer like hexamer or tetramer made up of two and three dimers appear. As $\epsilon$ keeps increasing, some dimers rotate to adjust themselves and then constitute the lattice of integer vortices. Under an initial conditions similar to an spinor BEC vortices dynamics experiment, the appearance of disordered turbulence is found in the process of fractional vortex generation, which matches the experimental observation. While in the formation process of integer vortices, the appearance of grooves is predicted.
\end{abstract}

The description of the nonequilibrium dynamics of strongly interacting quantum many body systems remains one of the most challenging problems in theoretical physics. In such systems, e.g. in a rotating strongly coupled superfluid, quantized vortices play an important role and the the vortices formation dynamics is always of great interest to study. In weakly coupled systems, quantum vortices have been experimentally realized in single component rotating Bose-Einstein condensates (BECs) of ultracold atomic gases [1. The Gross-Pitaevskii (G$\mathrm{P})$ equations based on a weakly coupled mean-field theory perfectly simulate condensation behaviors and quantized vortices in BECs at extremely low temperatures 2 5]. There are several experiments which are described by weakly coupled theories, such as superfluid He [6, 7], ultracold atoms 8 8 , and Fermi superfluids [1]. However, we need to go beyond weakly coupled theories to describe a strongly coupled BECs at a finite temperature, which is still a challenging problem [12. For decades, the anti-de Sitter(AdS)/conformal field theory(CFT) correspondence [13 16] or holography has been proved to be a unique "first principle" method to study equilibrium strongly coupled quantum systems at a finite temperature or zero temperature, by solving their dual weakly coupled gravity theory [17, 18. Also, the holographic method has successfully simulated the dynamics of a strongly coupled system far from equilibrium [19 24], then it is a perfect method to study the vortex formation dynamics and equilibrium state in a rotating single component strongly coupled BECs [25, 26, and also in the vortex for superconductor in a magnetic field $[27+29$.

Rather than a single component BEC, the twocomponent BECs of ${ }^{87} \mathrm{Rb}$ with the internal coherent
(Rabi or Josephson) coupling were also experimetally realized by the JILA group [30, 31]. With the development of study of vortices in ultracold atomic gases, many attentions have been paid to phenomena of vortices in multi-component condensations 3256 . In contrast to single-component BECs where only hexagonal Abrikosov lattice can form, a large number of lattice structures have been found in the two-component BECs. Such lattice structures are also expected in unconventional superconductors with multiple gap functions. When the intercomponent coupling is gradually increased, one observe transformations from the Abrikosov's hexagonal lattices to square lattices, stripe lattices and sheet lattices [34, 35]. Experimentally, a square lattice in two component spinor BEC has been found [57 and, more recently, a special lattice structure has been found in the mixture of Bose-Fermi superfluid with different masses 58. One of alluring features in multi-component condensations is that quantized circulations for superfluids 32 55] or fluxes for superconductors [59 62] are not integervalued anymore but are rational or fractional. In the order-parameter field, an integer quantized vortex has a winding $\phi \rightarrow \phi+2 \pi$ while a fractional quantized vortex does a winding $\phi \rightarrow \phi+\pi$ when the order-parameter phase $\phi$ passes through a path around the vortex core 63. However, when the internal coherent coupling $\epsilon$ is added in two-component BECs of ${ }^{87} \mathrm{Rb}$ experiments [30, 31, two fractional vortices in different components form a vortex pair bound by a domain wall, as shown in FIG. 1. and richer lattice structures would appear as predicted theoritically [36, 38. Finally, the two vortices overlap and form a integer vortex when $\epsilon$ exceeds a critical value [38. 

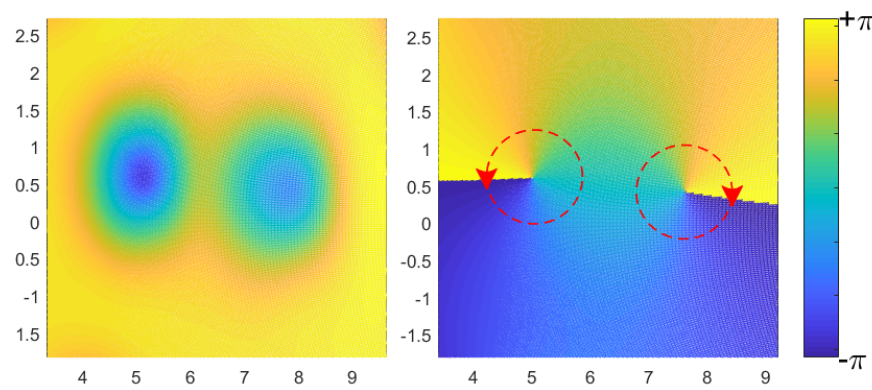

FIG. 1. (left): A vortex pair in a vortex lattice with $\eta=3$ and $\epsilon=0.005$. (right): The phase difference between the two components: $\phi(r)=\theta_{1}(r)-\theta_{2}(r)$ with the form that $\left\langle O_{1}\right\rangle=\left|\left\langle O_{1}\right\rangle\right| e^{i \theta_{1}}$ and $\left\langle O_{2}\right\rangle=\left|\left\langle O_{2}\right\rangle\right| e^{i \theta_{2}}$.

In this paper, we study the dynamic formation process of fractional and integer vortices in strongly coupled BECs made of two components and the dynamic crossover from fractional to integer vortices in strongly interacting systems, in the frame work of the AdS/CFT correspondence. We first use the time-dependent evolution of a single component to obtain a stable hexagonal Abrikosov lattice, then switch on the second component with the same chemical potential. We thus find a bizarre turbulence phenomenon and grooves in the coupling ranges of fractional vortices and integer vortices, respectively. These were not seen in the previous theoretical analysis 64, but are similar to the experiment [57. Two components behave independently like a single component BECs when the two couplings constants between the two components are vanishing, while once we turn on the coupling $\eta$, there will appear a rich lattice structure, as shown in FIG.2. Subsequently turning on $\epsilon$, the vortices in different components will form dimers. In the dynamic evolution, we see the autonomous arrangement of the dimers in the minimum free energy state, which is confirmed in FIG. 5 shown in 65, finally forming a lattice structure of integer vortices, as shown in FIG. 3 ,

The holographic model is constructed by a dual of an asymptotically AdS spacetime in three spatial dimensions with the quantum conformal field $\left(\mathrm{AdS}_{3+1} / \mathrm{CFT}_{2+1}\right)$. As found in Ref. [66, to construct a quantum field theory on the AdS boundary of two spatial dimensions, a complex scalar $\Psi(x)$, carrying charge $q$ under a global $U(1)$ symmetry is proposed to live in the $\mathrm{AdS}_{4}$ black hole. Let $j_{\mu}(x)$ denote the conserved current operator of this global $U(1)$ symmetry. To induce a superfluid condensate for $\Psi$, we will turn on a chemical potential $\mu$ for the $U(1)$ charge. For sufficiently large $\mu$, we expect $\Psi$ to develop a nonzero expectation value on the boundary when the temperature falls below a critical temperature $T_{c}$, spontaneously breaking the global $U(1)$ symmetry and driving the system into a superfluid phase. a) $\Omega=0.1 \eta=4 \epsilon=0$

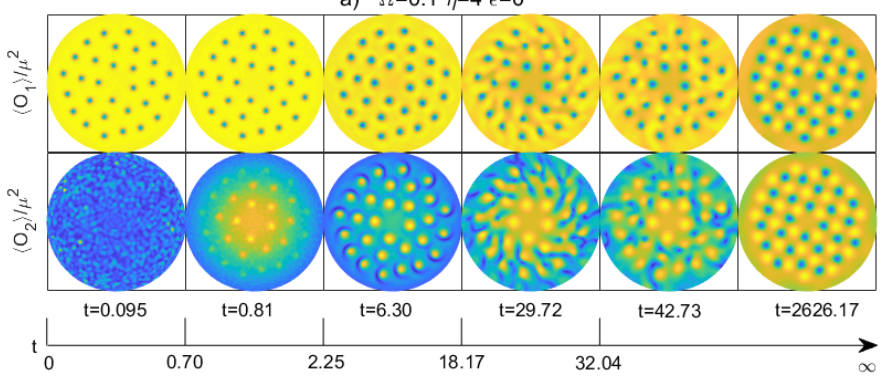

b) $\Omega=0.1 \eta=4 \epsilon=0.008$

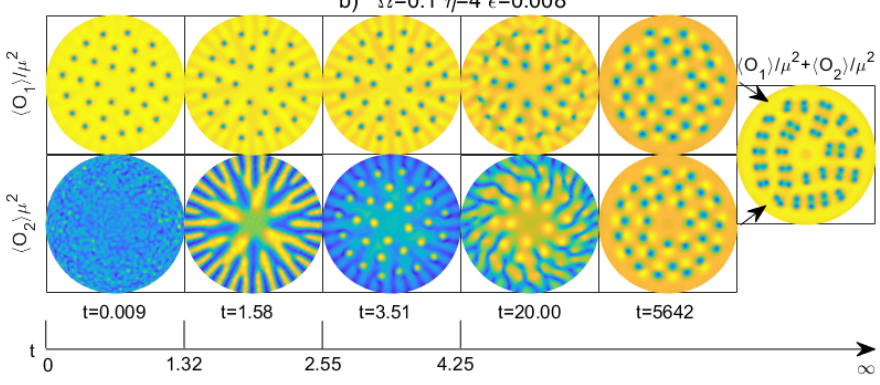

c) $\Omega=0.1 \eta=4 \epsilon=0.1$

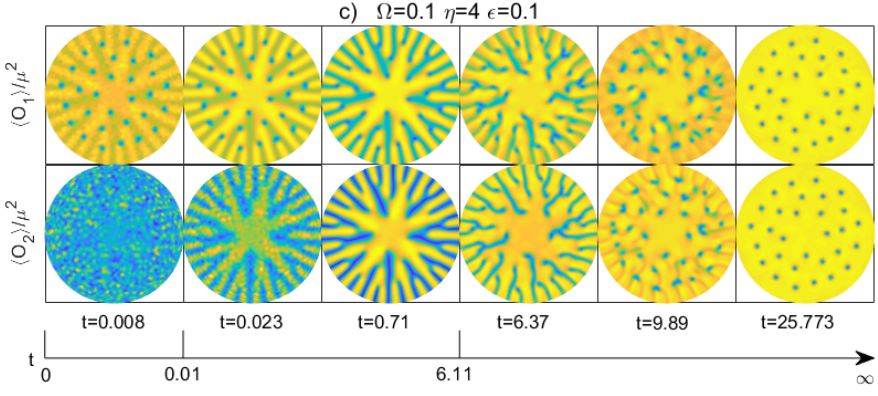

FIG. 2. The dynamics for three different vortex phases formation. (a) fractional vortices without internal coherent coupling, (b) dimers, (c) integer vortices. .

To simulate a two component BECs with the same chemical potentials, there are two complex bulk charged scalar fields $\Psi_{1}$ and $\Psi_{2}$, with charge $q$ and the mass $m_{j}$, separately coupled with two $U(1)$ gauge fields $A_{\mu(1,2)}$, thus yeilding two chemical potentials $\mu_{j}$. We then have the bulk action

$S=\int d^{4} x \sqrt{-g}\left[-\sum_{j=1}^{2}\left(\frac{1}{4} F_{j}^{2}+\left|D_{j} \Psi_{j}\right|^{2}+m_{j}^{2}\left|\Psi_{j}\right|^{2}\right)+V\right]$

The inter-component coupling potential between the two charged scalar fields takes the form

$$
V\left(\Psi_{1}, \Psi_{2}\right)=\epsilon\left(\Psi_{1} \Psi_{2}^{*}+\Psi_{1}^{*} \Psi_{2}\right)+\eta\left|\Psi_{1}\right|^{2}\left|\Psi_{2}\right|^{2},
$$

where $F_{j \mu \nu}=\partial_{\mu} A_{j \nu}-\partial_{\nu} A_{j \mu}, D_{j \mu}=\partial_{\mu}-i q A_{j \mu}$ with $q$ the charge. Here, $\eta$ is the intercomponent coupling coefficient and $\epsilon$ is the internal coherent (Rabi) coupling 

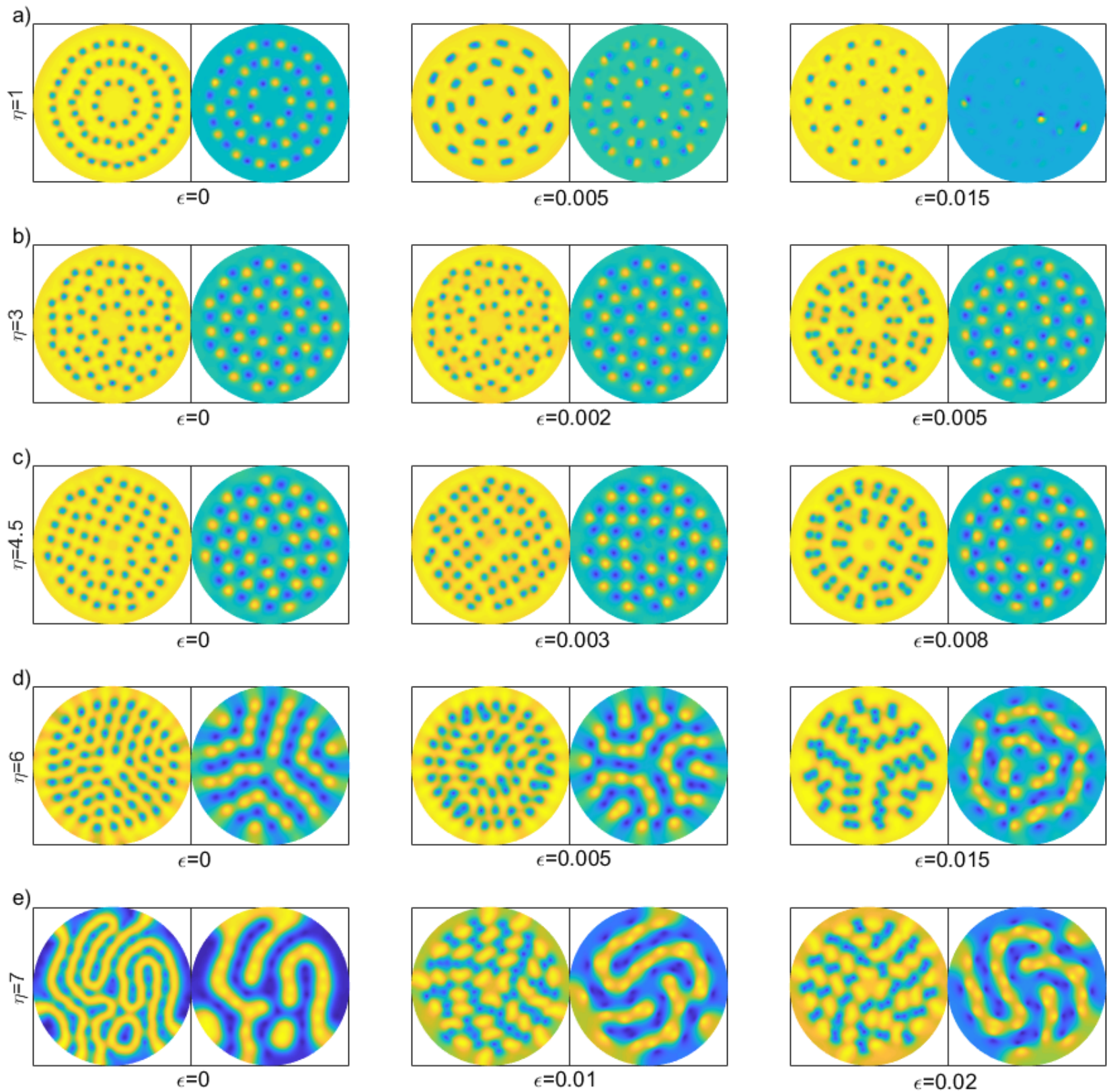

FIG. 3. The final equilibrium fractional and integer vortices by changing different $\epsilon$ with fixed $\eta$ after the formation of twocomponent vortex lattices. In all subfigures the left is a plot of the density profile of the conedensate $n=\left|\left\langle O_{1}\right\rangle\right|+\left|\left\langle O_{2}\right\rangle\right|$, the right panel is a figure of $\left|\left\langle O_{1}\right\rangle\right|-\left|\left\langle O_{2}\right\rangle\right|$ that displays $\left\langle O_{1}\right\rangle$ and $\left\langle O_{2}\right\rangle$ vortices, respectively, where blue parts represent vortices of $\left\langle O_{1}\right\rangle$ while yellow parts represent vortices of $\left\langle O_{2}\right\rangle$. The values of the coupling coefficient $\eta$ and $\epsilon$ are shown in the left and bottom, respectively, of each case.

coefficient. Notice that the two components are decoupled when $\eta=\epsilon=0$, the equations of motion and the numerical scheme can be found in [65]. This situation is different from that of the model proposed in Ref. 67, where the two components are always coupled even when both the coupling constants vanish, the vortex diagram of the model was studied and no fractional vortices were found 68 .

In FIG. 2, we show the evolution processes of the formation of vortex lattices from the holographic model Eq. 1. when $\mu_{1}=\mu_{2}>\mu_{c}$. We set the rotation speed to 0.1 for all dynamic processes. We first turn off the 
$\left\langle\mathrm{O}_{2}\right\rangle$ component by the vanishing condition for $\Psi_{2}$ in the dynamical process, which turns the kinetic equation into a single component equation like that in Ref. 66. We can see that the $\left\langle O_{1}\right\rangle$ component forms a hexagonal lattice like the first image of $\left|\left\langle O_{1}\right\rangle\right|$ in FIG. 2 (a). Then we give the $\left\langle\mathrm{O}_{2}\right\rangle$ component a slight perturbation, turn off the vanishing condition for $\Psi_{2}$ and also increase the $\eta$. Different evolution processes are illustrated in different $\epsilon$ values, such as the three subfigures in FIG. 2. We take the $\eta=4$, and take $\epsilon=0, \epsilon=0.008$ and $\epsilon=0.1$ in subfigures (a), (b), (c), respectively, to represent the fractional vortex range of the non-attractive interaction, dimer range and integer vortex range, respectively.

In the range $0<t<0.7$ of fractional vortices, the $\left\langle O_{2}\right\rangle$ has no change. On the other hand, when $0.7<t<2.25$, a condensation appears at the corresponding position of $\left\langle O_{1}\right\rangle$ vortices, which reflects the separation of the two components. When $2.25<t<6.3$, semicircular ripples appear around the condensation, and the direction of the ripples are opposite to that of rotation, which can be seen visually in the movie in the Supplemental Material. As evolution continues, these ripples rotate quickly and gradually spread out, forming a disorderly turbulence. This turbulence extends to the center of rotation and eventually vortices form directly in the turbulence when $t=32.04$. Both the turbulence and the vortices forming in it are separated from the vortices of $\left\langle O_{1}\right\rangle$. Over time, vortices of the two components form a square lattice in the end.

In the range of integer vortices, different phenomenon happens. In a very short time of about $t=0.01$, the two components respond. Exactly the same grooves form in the two components when $0.01<t<6.11$. These grooves gradually shrink until they form a complete superimposed integer vortex.

When $\epsilon$ is in the range of dimers, that is, the range between non-attractive interactions and integer vortices, we find that the vortex evolution process is like a combination of the two previous processes. The grooves and turbulence appear in the front and back stages, respectively. We infer that this is because in the initial stage, $\left\langle\mathrm{O}_{2}\right\rangle$ has fewer components, which is difficult to condense at the position of the $\left\langle O_{1}\right\rangle$ vortices, and so the separation cannot be shown. This indicates that the turbulence is caused by the separation interaction.

Next we investigate the dynamic evolution from fractional to integral vortices by gradually increasing $\epsilon$, as shown in FIG. 3. Subfigures (a,b), (c), (d) and (e) represent hexagonal, square, stripe and sheets lattices, respectively. Because the dividing line of multi-dimer/dimer and fractional/integer vortex lattices are approximatively linear between $\eta$ and $\epsilon$, it is convenient to use the ratio relation between $\eta$ and $\epsilon$ to discuss the process. When $0<\epsilon / \eta<0.00096$ and $3<\eta<5.5$, we find that multidimer bound states appear. When $\eta$ takes values corresponding to hexagonal lattices, hexamers composed of

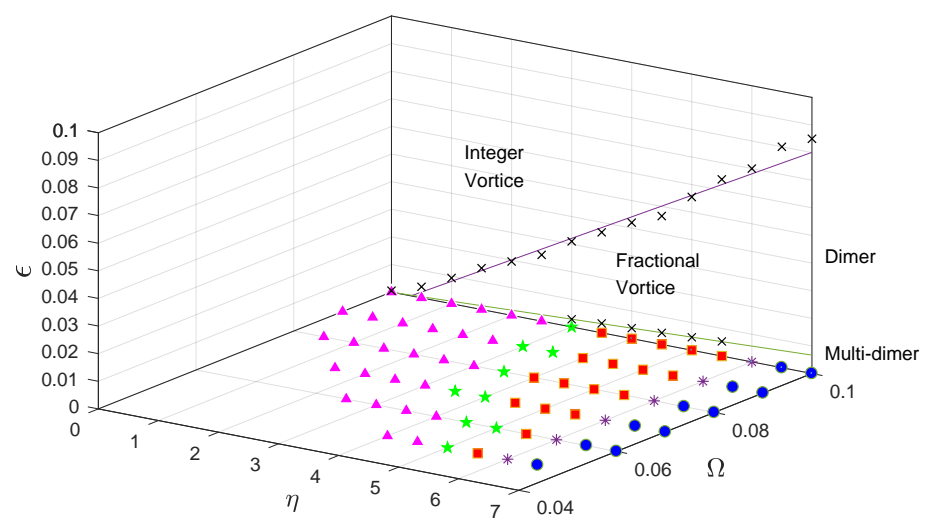

FIG. 4. (Bottom): the $\Omega-\eta$ phase diagram of the vortex states, where $\triangle, \star, \square, \times, \circ$, denote triangular lattices, triangular tetragonal cross lattices, square lattices, stripe vortices and vortex sheet lattices, respectively. (Side): the $\epsilon-\eta$ phase diagram with the boundary between fractional and integer vortices: $\epsilon=0.012 \eta-0.0039$ and the boundary between dimers and multi-dimers $: \epsilon=0.00096 \eta-0.000047$

three dimers form, as shown in middle part of FIG. 3(b). If $\eta$ is within the square lattice range, each bound state is composed of four vortices making up tetramers, as shown in the middle part of FIG. 3(c). However, different from the G-P equation, there are no multi-dimers when $\eta<3$ in our model. On the other hand, the phase separation for $\eta>5.5$ always prevents the formation of multi-dimer bound states for extremely low value of $\epsilon / \eta$. When $0.00096<\epsilon / \eta<0.012$, multi-dimers split into dimers. We find that dimers rotate to adjust themselves to meet the minimum energy requirements, which can be easily observed by comparing the middle and right figure in FIG. 3(b), (c), (d), (e). This rotation behavior also results in rich rotational symmetric lattice structures. While for $5.5<\eta$, the free energy is minimized when vortices of the same component are close to each other, forming stripe or sheet lattice, so for $\epsilon$ in the range of the non-integer vortices, there is always a phase separation. As $\epsilon$ continues to increase to the condition $\epsilon / \eta>0.012$, vortices in different components overlap to form an integer vortex lattice, as shown in the right figure of FIG. 3(a), where $\left|\left\langle O_{1}\right\rangle\right|-\left|\left\langle O_{2}\right\rangle\right|$ is almost zero.

By organizing the results, we obtain the phase diagram of fractional and integer vortex lattices with $0.05<\Omega<$ $0.1,0<\eta<7$ and $0<\epsilon<0.1$. The results are illustrated in FIG. 4 . The bottom phase diagram of $\Omega-\eta$ is similar as that in the G-P equations [34] and a simplified holographic model [68]. When we take $\Omega=0.1$ as specified above, by increasing $\eta$ from 0 , we can see hexagonal lattices $(0<\eta<3)$, square lattices $(3<\eta<5.5)$, stripe lattices $(5.5<\eta<6.5)$ and eventually sheet lattices $(6.5<\eta)$. When $\epsilon$ continues to increase, two vortices in each of the two components couple to form a dimer, and 
when $\eta$ is in the range of $(3-5.5)$, three or two dimers would further couple to form multi-dimers, such as hexamers and tetramers. By fitting the boundary between dimer and multi-dimer and between fractional and integer vortices, we can see two linear relationship between $\eta$ and $\epsilon$, similar to the results from the G-P equations [38.

In summary, with the power of the AdS/CFT correspondence, we have been able to access to the strongly coupled dynamics of a two component BEC by solving the classical gravitational equations in the bulk AdS space. A unique phenomenon of turbulence and grooves during the generation of fractional and integer vortex lattices have been observed. By comparing the generation process of different parameters, we have found that the turbulence is related to the separation interaction. In addition, we have studied the evolution process from the fractional vortex lattice to the integer vortex lattice with the increase of the internal coherent coupling $\eta$. We have found many differences with the results from the G-P equation, including the criticality of the multi-dimer, the rotation of dimers and rich lattice structures with slight differences.

Acknowledgements. - This work is supported by the National Natural Science Foundation of China (under Grant No. 11675140). This work of MN was supported in part by the Ministry of Education, Culture, Sports, Science (MEXT)-Supported Program for the Strategic Research Foundation at Private Universities "Topological Science" (Grant No. S1511006), Japan Society for the Promotion of Science (JSPS) KAKENHI (Grant Numbers 16H03984 and 18H01217) and by a Grant-in-Aid for Scientific Research on Innovative Areas "Topological Materials Science" (KAKENHI Grant No. 15H05855) from MEXT of Japan.

[1] D. V. Freilich, D. M. Bianchi, A. M. Kaufman, T. K. Langin, and D. S. Hall, Science 329, 1182 (2010); S. Middelkamp, P. J. Torres, P. G. Kevrekidis, D. J. Frantzeskakis, R. CarreteroGonzalez, P. Schmelcher, D. V. Freilich, D. S. Hall, Phys. Rev. A 84 011605(R) (2011); R. Navarro, R. Carretero-Gonzalez, P. J. Torres, P. G. Kevrekidis, D. J. Frantzeskakis, M. W. Ray, E. Altuntas, and D. S. Hall, Phys. Rev. Lett. 110, 225301 (2013).

[2] F. Dalfovo, S. Giorgini, L. P. Pitaevskii and S. Stringari, Rev. Mod. Phys. 71, 463 (1999).

[3] L. Pitaevskii and S. Stringari "Bose-Einstein Condensation and Superfluidity," (Oxford University Press, 2003),

[4] C. J. Pethick, and H. Smith, "Bose-Einstein Condensation in Dilute Gases," (Cambridge University Press, 2008).

[5] A. L. Fetter, Rev. Mod. Phys. 81, 647 (2009).

[6] V.K. Tkachenko, Sov. Phys. JETP 22, 1282 (1966); 23, 1049 (1966); 29, 945 (1969).

[7] L.J. Campbell and R.M. Ziff, Phys. Rev. B 20, 1886 (1979).

[8] K.W. Madison, F. Chevy, W. Wohlleben, and J. Dalibard,
Phys. Rev. Lett. 84, 806 (2000).

[9] J.R. Abo-Shaeer, C. Raman, J.M. Vogels, and W. Ketterle, Science 292, 476 (2001).

[10] Y.J. Lin, R.L. Compton, K.J. Garcia, J.V. Porto, and I.B. Spielman, Nature (London) 462, 628 (2009).

[11] M.W. Zwierlein, J.R. Abo-Shaeer, A. Schirotzek, C.H. Schunck, and W. Ketterle, Nature (London) 435, 1047 (2005).

[12] Allan. Griffin, Tetsuro. Nikuni, Eugene. Zaremba, "BoseCondensed Gases at Finite Temperatures", Cambridge University Press, 2009.

[13] J. M. Maldacena, Adv. Theor. Math. Phys. 2, 231 (1998).

[14] S. S. Gubser, I. R. Klebanov, and A. M. Polyakov, Phys. Lett. B 428, 105 (1998).

[15] E. Witten, Adv. Theor. Math. Phys 2, 253 (1998).

[16] O. Aharony, S. S. Gubser, J. M. Maldacena, H. Ooguri and Y. Oz, Phys. Rept. 323, 183 (2000).

[17] J. Zaanen, Y. W. Sun, Y. Liu and K. Schalm, "Holographic Duality in Condensed Matter Physics," Cambridge University Press, 2015.

[18] M. Ammon and J. Erdmenger, "Gauge/gravity duality: Foundations and applications," Cambridge University Press, 2015.

[19] H. Liu and J. Sonner, arXiv:1810.02367 [hep-th].

[20] A. Adams, P. M. Chesler, and H. Liu, Science 341, 368 (2013).

[21] J. Sonner, A. del Campo and W. H. Zurek, Nature Commun. 6, 7406 (2015)

[22] M. J. Bhaseen, J. P. Gauntlett, B. D. Simons, J. Sonner, and T. Wiseman, Phys. Rev. Lett. 110, 015301 (2013).

[23] P. M. Chesler, A. M. Garcia-Garcia and H. Liu, Phys. Rev. X 5, 021015 (2015).

[24] H. B. Zeng, C. Y. Xia, W. H. Zurek and H. Q. Zhang, arXiv:1912.08332 [hep-th].

[25] C. Y. Xia, H. B. Zeng, H. Q. Zhang, Z. Y. Nie, Y. Tian and X. Liu, Phys. Rev. D 100, 061901 (2019).

[26] X. Li, Y. Tian and H. Zhang, arXiv:1904.05497.

[27] Kengo. Maeda, Makoto. Natsuume, Takashi. Okamura, Phys. Rev. D 81, 026002 (2010).

[28] Aristomenis. Donos, Jerome. P. Gauntlett, Christiana. Pantelidou, arXiv:2001.11510

[29] Gianni. Tallarita, Roberto. Auzzi, JHEP 01, 056 (2020).

[30] D. S. Hall, M. R. Matthews, J. R. Ensher, C. E. Wieman, and E. A. Cornell, Phys. Rev. Lett. 81, 1539 (1998).

[31] M. R. Matthews, D. S. Hall, D. S. Jin, J. R. Ensher, C. E. Wieman, E. A. Cornell, F. Dalfovo, C. Minniti, and S. Stringari, Phys. Rev. Lett. 81, 243 (1998); M. R. Matthews, B. P. Anderson, P. C. Haljan, D. S. Hall, M. J. Holland, J. E. Williams, C. E. Wieman, and E. A. Cornell, Phys. Rev. Lett. 83, 3358 (1999); B. P. Anderson, P. C. Haljan, C. E. Wieman, and E. A. Cornell, Phys. Rev. Lett. 85, 2857 (2000).

[32] D. T. Son and M. A. Stephanov, Phys. Rev. A 65, 063621 (2002).

[33] E. J. Mueller and T.-L. Ho, Phys. Rev. Lett. 88, 180403 (2002).

[34] K. Kasamatsu, M. Tsubota, and M. Ueda, Phys. Rev. Lett. 91, 150406 (2003).

[35] K. Kasamatsu and M. Tsubota, Phys. Rev. A 79, 023606 (2009).

[36] K. Kasamatsu, M. Tsubota and M. Ueda, Phys. Rev. Leet. 93, 250406 (2004).

[37] K. Kasamatsu, M. Tsubota, and M. Ueda, Int. J. Mod. Phys. B 19, 1835 (2005). 
[38] M. Cipriani and M. Nitta, Phys. Rev. Lett. 111, 170401 (2013).

[39] P. N. Galteland, E. Babaev, and A. Sudbø, Phys. Rev. A 91, 013605 (2015).

[40] A. Aftalion, P. Mason, and J. Wei, Phys. Rev. A 85, 033614 (2012).

[41] P. Kuopanportti, J. A. M. Huhtamäki, and M. Möttönen, Phys. Rev. A 85, 043613 (2012).

[42] K. Kasamatsu and M. Tsubota, Phys. Rev. A 79, 023606 (2009).

[43] M. Eto, K. Kasamatsu, M. Nitta, H. Takeuchi and M. Tsubota, Phys. Rev. A 83, 063603 (2011).

[44] M. Eto and M. Nitta, Phys. Rev. A 85, 053645 (2012).

[45] M. Eto and M. Nitta, EPL 103, no. 6, 60006 (2013).

[46] M. Cipriani and M. Nitta, Phys. Rev. A 88, 013634 (2013).

[47] D. S. Dantas, A. R. P. Lima, A. Chaves, C. A. S. Almeida, G. A. Farias and M. V. Milošević, Phys. Rev. A 91, 023630 (2015).

[48] K. Kasamatsu, M. Eto and M. Nitta, Phys. Rev. A 93, no. 1, 013615 (2016).

[49] M. Tylutki, L. P. Pitaevskii, A. Recati and S. Stringari, Phys. Rev. A 93, no. 4, 043623 (2016).

[50] N. V. Orlova, P. Kuopanportti and M. V. Milošević, Phys. Rev. A 94, 023617 (2016).

[51] L. Calderaro, A. L. Fetter, P. Massignan, P. Wittek, Phys. Rev. A 95, no. 1, 023605 (2017).

[52] M. Eto and M. Nitta, Phys. Rev. A 97, no. 2, 023613 (2018).

[53] B. M. Uranga and A. Lamacraft, Phys. Rev. A 97, 043609 (2018).

[54] M. Kobayashi, M. Eto and M. Nitta, Phys. Rev. Lett. 123, no. 7, 075303 (2019).

[55] M. Eto, K. Ikeno and M. Nitta, arXiv:1912.09014 [condmat.quant-gas].

[56] S. H. Shinn and U. R. Fischer, Phys. Rev. A 98, no. 5, 053602 (2018).

[57] V. Schweikhard, I. Coddington, P. Engels, S. Tung, and E.A. Cornell, Phys. Rev. Lett. 93, 210403 (2004).

[58] X.C. Yao, H.Z. Chen, Y.P. Wu, X.P. Liu, X.Q. Wang, X. Jiang, Youjin Deng, Y.A. Chen, and J.W. Pan, Phys. Rev. Lett. 117, 145301 (2016).

[59] E. Babaev, Phys. Rev. Lett. 89, 067001 (2002); E. Babaev, A. Sudbo, and N. W. Ashcroft, Nature (London) 431, 666 (2004); J. Smiseth, E. Smorgrav, E. Babaev, and A. Sudbo, Phys. Rev. B 71, 214509 (2005); E. Babaev and N. W. Ashcroft, Nat. Phys. 3, 530 (2007).

[60] J. Goryo S. Soma, and H. Matsukawa, Europhys. Lett. 80, 17002 (2007).

[61] A. Crisan, Y. Tanaka, D. D. Shivagan, A. Iyo, L. Cosereanu, K. Tokiwa, and T. Watanabe, Jpn. J. Appl. Phys. 46, L451-L453 (2007); Y. Tanaka, A. Crisan, D. D. Shivagan, A. Iyo, K. Tokiwa, and T. Watanabe, Jpn. J. Appl. Phys. 46, 134-145 (2007); A. Crisan, A. Iyo, Y. Tanaka, H. Matsuhata, D. D. Shivagan, P. M. Shirage, K. Tokiwa, T. Watanabe, T. W. Button, and J. S. Abell, Phys. Rev. B 77, 144518 (2008); J. W. Guikema, H. Bluhm, D. A. Bonn, R. Liang, W. N. Hardy, and K. A. Moler, Phys. Rev. B 77, 104515 (2008); L. Luan, O. M. Auslaender, D. A. Bonn, R. Liang, W. N. Hardy, and K. A. Moler, Phys. Rev. B 79, 214530 (2009).

[62] Y. Tanaka, H. Yamamori, T. Yanagisawa. T. Nishio. S. Arisaw, Physica (Amsterdam) C 538, 6 (2017).

[63] S. Autti et al, Phys. Rev. Lett. 117,255301 (2016).
[64] T. Kanai and M. Tsubota, J. Phys.: Conf. Ser. 969 012016 (2018).

[65] See Supplemental Material for the detailed equations of motion and numerical scheme.

[66] S.A. Hartnoll, C.P. Herzong, and G.T. Horowitz, Phys. Rev. lett. 101, 031601 (2008).

[67] W. Y. Wen, M. S. Wu and S. Y. Wu, Phys. Rev. D 89, 066005 (2014)

[68] W. C. Yang, C. Y. Xia, H. B. Zeng, and H. Q. Zhang, arXiv:1907.01908 [hep-th]. 


\section{SUPPLEMENTAL MATERIAL}

\section{Equation of motion and numerical scheme}

The model proposed in Eq. 1 is living in a black hole whose metric in Eddington coordinate can be written as

$$
d s^{2}=\frac{\ell^{2}}{z^{2}}\left(-f(z) d t^{2}-2 d t d z+d r^{2}+r^{2} d \theta^{2}\right)
$$

in which $\ell$ is the AdS radius, $z$ is the AdS radial coordinate of the bulk and $f(z)=1-\left(z / z_{h}\right)^{3}$. Thus, $z=0$ is the AdS boundary while $z=z_{h}$ is the horizon; $r$ and $\theta$ are the radial and angular coordinates. The Hawking temperature is $T=3 /\left(4 \pi z_{h}\right)$. Without loss of generality we can rescale $q=\ell=z_{h}=1$. The probe limit is adopted in the paper by assuming that the matter fields do not affect the gravitational fields, as long as one neglects the effect of the superfluid condensate on the normal component of the fluid [1. This is the same as the G-P equations where the normal component is not considered in the model. The equations of motion are simply

$$
\begin{gathered}
d_{\nu} F_{j}^{\mu \nu}=J_{j}^{\mu} \\
\left(-D_{j}^{2}+m_{j}^{2}+\epsilon+\eta\left|\Psi_{k}\right|^{2}\right) \Psi_{j}=0
\end{gathered}
$$

where $j=1, k=2$ or $j=2, k=1$.

Under the standard holographic dictionary, the bulk scalar field $\Psi(r, \theta, z)$ can be mapped to a scalar operator $\Psi^{i}(r, \theta)$ of a quantum field theory at the boundary and $A_{\mu}(r, \theta, z)$ can be mapped to the conserved current $J^{\mu}(r, \theta)$. The axial gauge $A_{z}=0$ is adopted as in Ref. [1. Near the boundary, the general solutions take the asymptotic form as $A_{j \nu}=a_{j \nu}+\mathcal{O}(z), \partial_{z} A_{j \nu}=b_{j \nu}+\mathcal{O}(z)$, $(j=1,2)$. The coefficients $a_{j r, j \theta}$ can be regarded as the superfluid velocity along $r, \theta$ directions while $b_{j r, j \theta}$ as the conjugate currents [2]. $a_{j t}$ corresponds to the charge density $\rho$ of the field theory, while $b_{t}$ is the chemical potential $\mu$ of which is inversely proportional to the dimensionless temperature $T \propto 1 / \mu$. The $U(1)$ symmetry is spontaneously broken below the critical temperature, $T_{c}$, so as to increase the $\mu$ to a critical $\mu_{c}$, then the BECs form. In the broken phase, the field $\Psi$ has an non-zero solution whose expansion coefficient on the boundary is the expectation value of the dual operator in the field theory.
By choosing that $m_{1}^{2}=m_{2}^{2}=-2$ with $\Delta=2$, one can obtain $\partial_{z} \Psi_{j}=\Psi_{j}^{1}+\mathcal{O}(z), \partial_{z z} \Psi_{j}=2 \Psi_{j}^{2}+\mathcal{O}(z)$, where $\Psi_{j}^{1}$ is a source term which is always set to be zero in the spontaneous symmetry broken phase, $\Psi_{j}^{2}$ is the vacuum expectation value $\left\langle O_{j}\right\rangle$ of the dual scalar operator, which is non-vanishing in the broken phase.

The equations of motion (EoMs) are solved numerically by the Chebyshev spectral method in the $z, r$ direction, while the Fourier decomposition is adopted in the $\theta$ direction. The radius of the boundary disk is set as $r=R=20$. The Neumann boundary conditions are adopted both at $r=R$ and $r=0, \partial_{r} h_{i}=0$ where $h_{i}$ represents all the fields except $a_{j \theta}$. The rotation is introduced by imposing the angular boundary condition as $a_{j \theta}=\Omega r^{2}$ [3], where $\Omega$ is the constant angular velocity of the disk.

From the numerical simulation of the non-rotating state with the Newton-Raphson method we found that $\mu_{c} \sim 4.07$ when $\epsilon=\eta=0$, so the dimensionless critical temperature is $T_{c}^{0}=\frac{3}{4 \pi \mu_{c}(0,0)}=0.0587$. With the fixed $\mu=6$, we obtain the uniform solution in the non-rotating state with the Newton-Raphson method, which can be served as the initial value of dynamic simulation. Begin with this initial uniform solution, the time evolution is simulated by the fourth order Runge-Kutta method. By setting the $\eta, \epsilon$ and $\Psi_{2}$ to zero, we obtain a single component EoMs, which end up with a hexagonal lattice. Then, given a finite value of $\eta$ and $\epsilon$ and a perturbation of $\Psi_{2}$, one can obtain the evolution of two-component vortex lattices similar to the experiment [4.

\section{Free Energy}

At a fixed temperature, the free energy $F$ can be computed from the renormalized on-shell action $S_{\text {ren }}$ with two parts $S_{\text {o.s. }}$ and $S_{\text {c.t. }} . F=T S_{\text {ren }}=T\left(S_{\text {o.s. }}+S_{\text {c.t. }}\right)$, in which $S_{o . s}$. is the bare on-shell action obtained by subtracting the equation of motions from Eq. (1) while $S_{c . t .}=-\left.\sum_{j=1}^{2} \int d t d r d \theta \sqrt{-\gamma} \Psi_{j} \Psi_{j}^{\star}\right|_{z=0}$ is the term to remove the divergence near the boundary $z=0$, where $\gamma$ is the determinant of the reduced metric. Then, one can obtained the form of the renormalized on-shell action that

$$
\begin{array}{r}
S_{r e n}=\frac{1}{2} \sum_{j=1}^{2}\left\{-\left.\int d t d z d \theta\left[\frac{1}{r} A_{\theta j} \partial_{r} A_{\theta j}\right]\right|_{r=R}+\int d t d r d \theta\left[r\left(-a_{t j} b_{t j}+\frac{1}{r^{2}} a_{\theta j} b_{\theta j}+\Psi_{j}^{1 \star} \Psi_{j}^{2}+\Psi_{j}^{2 \star} \Psi_{j}^{1}\right)\right\}\right. \\
\left.+\frac{i q}{2} \sum_{j=1}^{2} \int d t d z d r d \theta\left[\frac{r}{z^{4}} A_{\mu j}\left(\Psi_{j}^{\star} \partial^{\mu} \Psi_{j}-\Psi_{j} \partial^{\mu} \Psi_{j}^{\star}\right)-2 i q A_{j}^{\mu}\left|\Psi_{j}\right|^{2}\right]\right] \\
+\int d t d z d r d \theta\left[\epsilon\left(\Psi_{1} \Psi_{2}^{\star}+\Psi_{1}^{\star} \Psi_{2}+\left|\Psi_{1}\right|^{2}+\left|\Psi_{2}\right|^{2}\right)+3 \eta\left|\Psi_{1}\right|^{2}\left|\Psi_{2}\right|^{2}\right]
\end{array}
$$


In the Fig 5 we calculated the free energy $\left(F-F_{n}\right) / T$ of a dynamic process with parameter that $\Omega=0.1, \eta=4$ and $\epsilon=0$, in which $F_{n}$ is the free energy in the normal state, i.e., $\Psi_{j}=0$.. It can be seen that after $t=500$, the free energy reaches its lowest point and doesn't fluctuate any more. Then, we can say that after this time, the system reaches equilibrium.

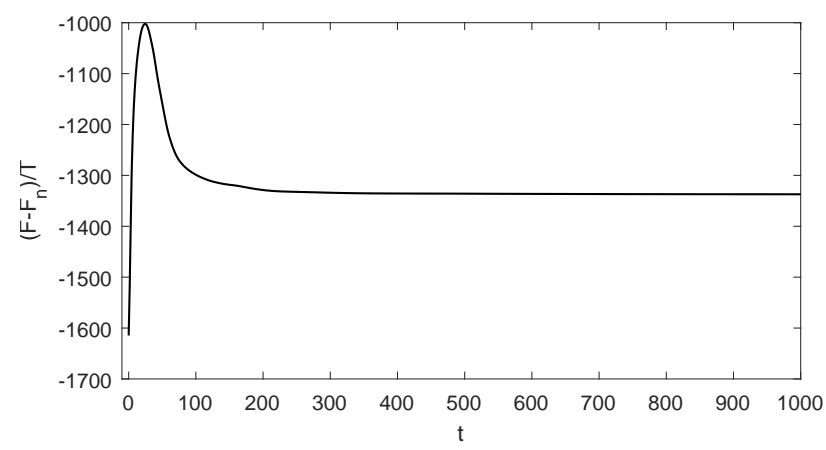

FIG. 5. The time evolution of rescaled free energy with $\Omega=$ $0.1, \eta=4$ and $\epsilon=0$.
[1] A. Adams, P. M. Chesler, and H. Liu, Science 341, 368 (2013).

[2] M. Montull, A. Pomarol and P. J. Silva, Phys. Rev. Lett. 103, 091601 (2009).

[3] O. Domenech, M. Montull, A. Pomarol, A. Salvio and P. J. Silva, JHEP 1008, 033 (2010).

[4] V. Schweikhard, I. Coddington, P. Engels, S. Tung, and E.A. Cornell, Phys. Rev. Lett. 93, 210403 (2004). 